Commun. math. Phys. 38, 173-193 (1974)

(C) by Springer-Verlag 1974

\title{
Stability and Equilibrium States
}

\author{
Rudolf Haag, Daniel Kastler, and Ewa B. Trych-Pohlmeyer \\ II. Institut für theoretische Physik der Universität, Hamburg, \\ Federal Republic of Germany, and \\ Département de Physique, Centre Universitaire de Marseille-Luminy, Marseille, France
}

Received May 20, 1974

\begin{abstract}
For an infinite dynamical quantum system idealized as a $C^{*}$-algebra acted upon by time-translations automorphisms in an asymptotically abelian way, we propose to characterize equilibrium states by the three properties of stationarity, stability for local perturbations of the dynamics, and relative purity. We show that a state with these properties either gives rise to a one-sided energy spectrum or is a KMS- (i.e. essentially a limit Gibbs-) state.
\end{abstract}

\section{Introduction}

The problem of characterizing equilibrium states in statistical mechanics is traditionally treated in the following steps:

1) One argues that the equilibrium value of an observable (in an isolated system of finite size) corresponds to the average value in a microcanonical ensemble of such systems.

2) One considers the thermodynamic limit, increasing the size of the system to infinity. It is in this limit that the fluctuations within the microcanonical ensemble become zero for intensive quantities and the thermodynamic laws become true for the individual system. Moreover, in this limit various ensembles (such as the microcanonical, the canonical, the uniform ensemble) become equivalent in the sense that they all give the same expectation value for local observables.

Let us call the expectation functional over the local observables which is obtained in the thermodynamic limit from any of the above mentioned ensembles a "limit Gibbs' state". The limit Gibbs states of a quantum system have an interesting property, first pointed out by Kubo [1] and by Martin and Schwinger [2] which may serve to compute these states without considering finite systems and limiting procedures. The Kubo-Martin-Schwinger (KMS)-condition, formulated algebraically in [3], has been the subject of much study in recent years by physicists and mathematicians.

From the point of view of physics, this work has strengthened the belief that the KMS-condition gives indeed an adequate characterization of equilibrium states in an infinite quantum system. On 
the other hand, it was realized that this condition is intimately related to the mathematical theory of modular automorphisms of operator algebras developed by Tomita and Takesaki (compare e.g. [4-7]).

Of course, a basic concept like that of an equilibrium state should ultimately be defined in simple physical terms. We propose to do that here for an infinite quantum system. Specifically we shall consider the following as the three defining properties of an equilibrium state:

(i) Stationarity.

(ii) Stability under local perturbations of the dynamics.

(iii) Relative purity.

Starting from these requirements and some natural assumptions about the dynamical system, we give a simple and direct derivation of the KMS-condition ${ }^{1}$.

We shall do this on two levels. A mathematically precise presentation of the argument will be given in Sections II to IV. For the benefit of physicists who do not like theorems and lemmas we shall use the remainder of this introduction for some remarks and, ultimately, a brief sketch of the main line of the argument in a less sophisticated language, disregarding all technical details and questions of rigour ${ }^{2}$.

Of the three requirements the first needs no comment, the third is more or less standard and it is needed to insure that the intensive quantities have sharp values (zero fluctuations).

The second requirement (stability) needs some elaboration. It is made to eliminate the assumption of ergodicity of the system. The connection may be seen in the following way. The intuitive arguments given by Willard Gibbs to justify the first of the above mentioned steps in the traditional approach have led to the development of ergodic theory. Roughly speaking, a system may be called ergodic if, apart from the known general conservation laws (linear and angular momentum, energy, particle numbers) there are no other relevant constants of motion $^{3}$. Here the difficulties in the classical and quantum mechanical cases appear at quite different stages. The case of a finite quantum system

1 In the derivation of the KMS-condition we shall use instead of the requirement iii) the stronger assumption that correlations between local quantities at different times vanish sufficiently fast as the time differences go to infinity.

2 Pure mathematicians rebuked by physical considerations may read Sections II through IV as a logically self-consistent paper.

3 The term "ergodic" has been used in several different senses. What we mean here is "ergodicity of the system" which is a property of the dynamical law alone in contradistinction to "ergodicity of a state" which in standard mathematical terminology is synonymous with the requirements i) and iii) above. Every system has ergodic states, but for the purposes of statistical mechanics the role of ergodic theory is to show that specific states (in the case of classical finite systems the microcanonical ensembles, in infinite quantum systems the limit Gibbs states) are ergodic. In other words, the system may be called ergodic if our requirement ii) is redundant in the definition of equilibrium states. 
is essentially trivial in this respect. The energy spectrum is discrete and will ordinarily not have any accidental degeneracy. The difficulty appears there only in the thermodynamic limit due to the increasing level density (almost degeneracy). Let us nevertheless, by way of analogy, consider a finite quantum system with a spectrum which does have an accidental degeneracy (corresponding to nonergodicity) ${ }^{4}$. Suppose that the Hamiltonian $H$ is changed by a small perturbation to $H^{\prime}=H+\lambda V$. Schrödinger's perturbation theory tells us that only certain vectors in the degeneracy space will join smoothly to the eigen-vectors of the perturbed Hamiltonian. The selection of those specific eigen-vectors of $H$ which go over continuously into eigen-vectors of $H^{\prime}$ depends on the perturbation $V$. If we want a stationary state of $H$ to energy $E$ which changes continuously under any small perturbation (or, as we might say, a stationary state which is stable under any small perturbation), then we cannot take a pure state (eigenvector), but have to use a density matrix which is the (normalized) unit matrix in the degeneracy space of the eigenvalue $E$. In other words, no matter whether or not there exist any constant of motion, a stationary state stable under arbitrary small perturbations is always given by a density matrix which is a function of $H$.

On the other hand, as mentioned at the very beginning, it is not important to specify precisely the functions of the Hamiltonian which we take as density matrices for the finite system. The set of relatively pure states arising in the thermodynamic limit will be unaffected (coalescence of different ensembles to one limit Gibbs state). These two facts taken together make it understandable why limit Gibbs states are singled out by the requirements (i)-(iii).

An illustrative example is a system of non interacting particles. The limit Gibbs states of this system describe an ideal gas. The system is highly non ergodic. There are many constants of motion and, correspondingly, many relatively pure, stationary states which are not limit Gibbs states ${ }^{5}$. In text-books of statistical mechanics, this fact is well recognized and it is pointed out that one should imagine the addition of a little dust to provide a mechanism for transforming the momentum distribution of the particles into the stable one. The precise implementation of this idea in the case of an infinite system of non interacting Fermions is described in the appendix. For more details see [8].

We come now to the sketch of the general case. We may consider a (uniformly closed) algebra $\mathfrak{A}$ of bounded operators acting on a Hilbert space $\mathscr{H}$. The self adjoint elements of $\mathfrak{A}$ represent (bounded) quasilocal

${ }^{4}$ For simplicity we shall suppress the universal constants of motion and speak in the following of degeneracy instead of accidental degeneracy.

${ }^{5}$ E.g. all translationally invariant states with vanishing interparticle correlations but arbitrary single-particle momentum distribution. 
observables in the infinitely extended medium. The equilibrium state $\omega$ in which we are interested will be represented by a state vector $\Omega \in \mathscr{H}$. Note that we take a vector and not a density matrix. This is always possible; the price one pays if $\omega$ is not pure is the reducibility of $\mathfrak{A}$ (i.e. there will exist in that case non trivial operators on $\mathscr{H}$ which commute with all operators from $\mathfrak{A})$. Still we can approximate every vector in $\mathscr{H}$ arbitrarily well by applying an operator from $\mathfrak{A}$ to $\Omega$. Therefore all vectors in $\mathscr{H}$ represent states which may be regarded as quasilocal excitations of the state $\omega$.

Acting in $\mathscr{H}$ we have a Hamiltonian operator $H$ with the Heisenberg equations of motion for observables

and the relation

$$
\frac{\partial A}{\partial t}=i[H, A]
$$

$$
H \Omega=0
$$

expressing the fact that $\omega$ is stationary and that we have (arbitrarily) assigned to $\Omega$ the zero point in the $H$-spectrum. Of course, $H$ is a global (extensive) quantity, in general not affiliated with the algebra $\mathfrak{A}$ of quasilocal observables. It would also be slightly misleading to call $H$ the energy operator since the physical energy (the space integral of the local energy density) has infinite fluctuations which are subtracted out in the construction of $H$ (see e.g. [3]).

We denote the expectation value of $A \in \mathfrak{A}$ in the equilibrium state $\omega$ by $\omega(A)$ and the time translate of $A$, arising from the integration of $(1,1)$ by $A_{t}$ (in subsequent sections the notation $\alpha_{t}(A)$ will be used instead):

$$
\begin{aligned}
\omega(A) & =(\Omega, A \Omega), \\
A_{t} & =e^{i H t} A e^{-i H t} .
\end{aligned}
$$

Our first assumption concerns the "correlation functions" \langle\rangle$_{T}$ or "truncated expectation values" defined in the well known manner

$$
\left\langle A_{t_{1}} B_{t_{2}}\right\rangle_{T}=\omega\left(A_{t_{1}} B_{t_{2}}\right)-\omega(A) \omega(B) \quad \text { etc. }
$$

We assume that these functions die out when the time differences become large. This is essentially a consequence of the physical property that any two local observables measured at different times become more and more compatible when the time difference goes to infinity (asymptotic Abelianness in time) and of the requirement of relative purity for $\omega$ (see e.g. [9]). This assumption has several important consequences. First, for any $A \in \mathfrak{A}, A_{t}$ converges weakly to a multiple of the unit operator as $t \rightarrow \pm \infty$ :

$$
\lim _{t \rightarrow \pm \infty}\left(\psi, A_{t} \phi\right)=\omega(A)(\psi, \phi) ; \quad \psi, \phi \in \mathscr{H}
$$


Secondly, $H$ has only one proper eigenvector namely the vector $\Omega$, belonging to the eigenvalue zero. The rest of the spectrum of $H$ is continuous. Thirdly, the spectrum of $H$ is either one-sided (positive or negative semidefinite) or it is the whole real line (see Proposition 3). If the spectrum is one-sided the state $\omega$ is pure [10] and this corresponds to the zero temperature situation with which we shall not be concerned in this paper. Thus, in the case to be considered here, the $H$-spectrum is a continuum extending from $-\infty$ to $+\infty$ plus one discrete point, the zero.

Consider now the effect of a local perturbation of the dynamics, i.e. replace $H$ by

$$
H^{(\lambda, h)}=H+\lambda h
$$

where $h$ is a local, bounded observable and $\lambda$ a coupling constant. Let us compute the new stationary state $\omega^{(\lambda h)}$ by first order perturbation theory. Because of the reducibility of $\mathfrak{A}$, it is better to apply the perturbation theory not to the state vectors, but to expectation functionals. Writing

$$
\omega^{(\lambda h)}=\omega+\lambda \omega_{1}^{(h)}+\cdots
$$

we get for $\omega_{1}^{(h)}$ the conditions

$$
\begin{aligned}
\omega_{1}^{(h)}([H, A]) & =\omega([h, A]), \\
\omega_{1}^{(h)}(I) & =0 .
\end{aligned}
$$

(1.9) resulting from the normalization of $\omega^{(\lambda h)}$. Due to (1.6) the unique solution is

$$
\omega_{1}^{(h)}(A)=\lim _{T \rightarrow \pm \infty} i \int_{T}^{0} d t \omega\left[h, A_{t}\right] d t
$$

The two alternative expressions $(T \rightarrow \pm \infty)$ show that the unperturbed state $\omega$ has to satisfy the consistency condition

$$
\int_{-\infty}^{\infty} d t \omega\left(\left[h, A_{t}\right]\right)=0 \text {. }
$$

This condition is the analogue of the secular equation of the Schrödinger perturbation theory in the degenerate spectrum for our situation in which the discrete eigenvalue zero is "degenerate" with an overlapping continuous spectrum. Physically this condition results from the requirement that a small local perturbation should produce only a small, local change in the stationary state.

Let us consider now for $A, B \in \mathfrak{A}$ the two functions

$$
\begin{aligned}
& F_{A B}(t)=\omega\left(B A_{t}\right)-\omega(B) \omega(A), \\
& G_{A B}(t)=\omega\left(A_{t} B\right)-\omega(B) \omega(A)
\end{aligned}
$$

and denote their Fourier transforms by $\hat{F}_{A B}(E), \hat{G}_{A B}(E)$. The KMScondition for the state $\omega$ can be expressed as the statement that there exists a real parameter $\beta$ such that (see e.g. [11])

$$
\hat{F}_{A B}(E)=e^{\beta E} \hat{G}_{A B}(E) \quad \text { for all } \quad A, B \text {. }
$$


The consistency condition (1.11) is precisely this statement for $B=h$ and the special value $E=0$. If we require stability for all quasilocal perturbations the full condition (1.14) may be derived from (1.11) by the following trick assuming a sufficiently rapid decrease of the correlation functions. Replacing $A$ in (1.11) by $A^{(1)} A_{\tau}^{(2)}$ and $h$ by $h^{(1)} h_{\tau}^{(2)}$ and taking the limit $\tau \rightarrow \infty$ we obtain with $F^{(i)}=F_{A^{(i)} h^{(i)}}, i=1,2$

$$
\int_{-\infty}^{\infty} F^{(1)}(t) F^{(2)}(t) d t=\int G^{(1)}(t) G^{(2)}(t) d t
$$

Using the freedom to vary $A^{(1)}, A^{(2)}, h^{(1)}, h^{(2)}$ independently, one concludes that $\hat{F}_{A B}(E)=\phi(E) \hat{G}_{A B}(E)$, where $\phi(E)$ is a universal function, independent of the choice of $A$ and $B$. Repetition of the step leading from (1.11) to (1.15) and the use of reality and symmetry properties of the functions $\hat{F}, \hat{G}$ leads then to the result that $\phi(E)$ must be a real-multiplicative function, i.e. an exponential: that is the KMS-condition (1.14).

Finally we note that the KMS-condition is not only necessary but also sufficient for the stability of the state under local perturbations of the dynamics. This property of KMS-states has been proven by Araki in [12].

\section{Stability for Local Perturbations of the Dynamics}

Definition 1. We will call dynamical system $\{\mathfrak{U}, \alpha\}$ the pair of a $C^{*}$-algebra $\mathfrak{a}$ together with a strongly continuous group $t \in \mathbb{R} \rightarrow \alpha_{t}$ of automorphisms of $\mathfrak{A}$. Strong continuity of $\alpha$ is defined as the fact that the map $t \rightarrow \alpha_{t}(A)$ is continuous in norm for all $A \in \mathfrak{A}^{6}$.

$A$ stationary state $\omega$ of the dynamical system $\{\mathfrak{A}, \alpha\}$ is a state of $\mathfrak{A}$ such that $\omega \circ \alpha_{t}=\omega$ for all $t \in \mathbb{R}$.

Our terminology is motivated by the fact that in our case the physical system is an infinitely extended medium, the quasi-local observables of which are represented by the self adjoint elements $A \in \mathfrak{A}$, whilst the automorphism $\alpha_{t}$ corresponds to the time-translation by $t$.

The next proposition borrowed from Araki [13], describes the concept of quasi-local (or "inner") perturbations of the dynamics. (see also [15]) ${ }^{7}$.

${ }^{6}$ This is equivalent to the continuity of all numerical functions $t \rightarrow \phi\left(\alpha_{t}(A)\right), A \in \mathfrak{A}$, $\phi$ any state of $\mathfrak{A}$; see for instance Chapter IX in [14].

${ }^{7}$ Robinson defines the perturbed automorphism by the formula:

$$
\begin{gathered}
\alpha_{t}^{(h)}(A)=\sum_{n=0}^{\infty} \int d t_{1} \ldots d t_{n}\left[\alpha_{t_{1}}(h)\left[\alpha_{t_{2}}(h) \ldots\left[\alpha_{t_{n}}(h), A\right] \ldots\right]\right] \\
0<t_{1}<\cdots t_{n}<t .
\end{gathered}
$$


Proposition 1. Let $\{\mathfrak{U}, \alpha\}$ be a dynamical system and consider $a$ self adjoint element $h$ of $\mathfrak{U}$. The differential equation

$$
i \frac{d \Gamma_{t}^{(h)}}{d t}=\Gamma_{t}^{(h)} \alpha_{t}(h)
$$

(where the derivative is in the norm-sense) together with the initial condition

$$
\Gamma_{0}^{(h)}=I
$$

defines uniquely a continuous function $t \in \mathbb{R} \rightarrow \Gamma_{t}^{(h)} \in \mathfrak{A}$ with the following properties

(i) $\Gamma_{t}^{(h)}$ is given by the norm-convergent expansion

where

$$
\Gamma_{t}^{(h)}=\sum_{n=0}^{\infty} \Gamma_{t, n}^{(h)}
$$

$$
\Gamma_{t, n}^{(h)}=(-i)^{n} \int_{0}^{t} d t_{n} \int_{0}^{t_{n}} d t_{n-1} \ldots \int_{0}^{t_{2}} d t_{1} \alpha_{t_{1}}(h) \alpha_{t_{2}}(h) \ldots \alpha_{t_{n}}(h) .
$$

(ii) $\Gamma_{t}^{(h)}$ is unitary for all $t \in R$ with

$$
\Gamma_{t}^{(h)^{*}}=\Gamma_{t}^{(h)^{-1}}=\alpha_{t}\left(\Gamma_{-t}^{(h)}\right) .
$$

(iii) $\Gamma_{t}^{(h)}$ fulfills the "cocycle property"

$$
\Gamma_{s+t}^{(h)}=\Gamma_{s}^{(h)} \alpha_{s}\left(\Gamma_{t}^{(h)}\right) .
$$

(iv) Consequently $t \rightarrow \alpha_{t}^{(h)}$, given by

$$
\begin{aligned}
\alpha_{t}^{(h)}(A) & =\Gamma_{t}^{(h)} \alpha_{t}(A) \Gamma_{t}^{(h)^{*}} \\
& =\alpha_{t}\left(\Gamma_{-t}^{(h)} A \Gamma_{-t}^{(h)}\right) \quad t, \in R, A \in \mathfrak{U}
\end{aligned}
$$

is a strongly continuous one-parameter group of automorphisms of $\mathfrak{A}$. We say that $\alpha^{(h)}$ is obtained by "perturbing $\alpha=\alpha^{(0)}$ with the inner perturbation $h$. The latter is a perturbation of the Hamiltonian in the following sense:

(v) The element $A$ of $\mathfrak{A}$ is (norm-) differentiable for $\alpha$ if and only if it is norm-differentiable for $\alpha^{(h)}$ and the two derivatives are related by

$$
\left.\frac{d}{d t}\right|_{t=0} \alpha_{t}^{(h)}(A)=\left.i \frac{d}{d t}\right|_{t=0} \alpha_{t}(A)+[h, A]
$$

where we use the customary notation for a commutator

$$
[h, A]=h A-A h \text {. }
$$

Definition 2. Let $\omega$ be a stationary state of the dynamical system $\{\mathfrak{U}, \alpha\}$ and denote as above by $\alpha^{(h)}$ the one-parameter group of automorphisms of $\mathfrak{A}$ obtained by perturbing $\alpha$ with the inner perturbation 
$h=h^{*} \in \mathfrak{A}$. We say that $\omega$ is stable for the inner perturbation $h$ whenever there is a map $\lambda \rightarrow \omega^{(\lambda h)}$ from some neighbourhood of zero in $\mathbb{R}$ to the state space of $\mathfrak{U}$ such that

(i) $\omega^{(\lambda h)}$ is a stationary state of the perturbed dynamical system $\left\{\mathfrak{U}, \alpha^{(h)}\right\}$.

(ii) The map $\lambda \rightarrow \omega^{(\lambda h)}$ is weakly differentiable for $\lambda=0$

$$
\left(\text { with derivative }\left.\frac{d \omega^{(\lambda h)}}{d \lambda}\right|_{\lambda=0} \text { denoted } \omega_{1}^{(h)}\right)^{8} .
$$

If we have in addition that

(iii) the derivative $\omega_{1}^{(h)}$ lies in the normal folium of $\omega$ (i.e. is a normal form of the representation of $\mathfrak{U}$ generated by $\omega$ ) we say that $\omega$ is foliumstable for the inner perturbation $h$. Finally if $\omega$ is (folium-) stable for all perturbations $h=h^{*} \in \mathfrak{U}$ we say that $\omega$ is (folium-) stable for inner perturbations.

This definition formulates the physical requirement that persistent quasi-local perturbations of the dynamics do not essentially modify the equilibrium states of infinite systems. They merely cause gentle displacements of the equilibrium.

We note as an immediate consequence of its definition that the derivative $\omega_{1}^{(h)}$ vanishes on multiples of the unit.

The next proposition is a first step towards computing $\omega_{1}^{(h)}$ in terms of $\omega$ and $h$.

Proposition 2. Let $\omega$ be a stationary state of the dynamical system $\{\mathfrak{A}, \alpha\}$ stable for the inner perturbation $h=h^{*} \in \mathfrak{A}$, with $\omega_{1}^{(h)}$ as in the definition above. We have, for $A \in \mathfrak{U}$ and $S, T \in \mathbb{R}$

$$
\omega_{1}^{(h)}\left(\alpha_{T}(A)-\alpha_{S}(A)\right)=i \omega\left\{\left[h, \int_{S}^{T} \alpha_{t}(A) d t\right]\right\}
$$

where the integral in the right-hand side is a Bochner integral and [] denotes a commutator.

The proof is immediate from the two following lemmas.

Lemma 1. With $\omega$ as in the Proposition above we have for each $A \in \mathfrak{U}$ norm-differentiable for $\alpha$

$$
i \omega_{1}^{(h)}\left\{\left.\frac{d}{d t}\right|_{t=0} \alpha_{t}(A)\right\}+\omega(h A-A h)=0 .
$$

8 This notation is motivated by the fact that $\omega_{1}^{(h)}$ is the first term of an expansion in powers of $\lambda[15]$. 
Proof. Using (2.8) [see (v) of Proposition 1 above] and the fact that $\omega^{(\lambda h)}$ is invariant for $\alpha^{(\lambda h)}$ we have that, for each norm-differentiable $A \in \mathfrak{A}$,

$$
\begin{aligned}
0= & \omega^{(\lambda h)}\left(\left.i \frac{d}{d t}\right|_{t=0} \alpha_{t}^{(\lambda h)}(A)\right)=\omega\left(\left.i \frac{d}{d t}\right|_{t=0} \alpha_{t}(A)\right) \\
& +\lambda \omega(h A-A h)+\left\{\omega^{(\lambda h)}-\omega\right\}\left\{\left.i \frac{d}{d t}\right|_{t=0} \alpha_{t}(A)+\lambda(h A-A h)\right\}
\end{aligned}
$$

where the first term on the right hand side vanishes by the invariance of $\omega$ for $\alpha$. Hence

$$
\omega(h A-A h)+\frac{\omega^{(\lambda h)}-\omega}{\lambda}\left(\left.i \frac{d}{d t}\right|_{t=0} \alpha_{t}(A)+\lambda(h A-A h)\right)=0
$$

yielding (2.10) in the limit $\lambda \rightarrow 0$.

Lemma 2. With $\{\mathfrak{U}, \alpha\}$ a dynamical system and $A$ any element of $\mathfrak{U}$ the Bochner integral

$$
A_{S}^{T}=\int_{S}^{T} \alpha_{t}(A), \quad S, T \in \mathbb{R}
$$

defines an element $A_{T} \in \mathfrak{A}$ norm differentiable for $\alpha$ with derivative

$$
\left.\frac{d}{d t}\right|_{t=0} \alpha_{t}\left(A_{S}^{T}\right)=\alpha_{T}(A)-\alpha_{S}(A) .
$$

Proof. By the translation invariance of the Lebesgue measure

Thus

$$
\alpha_{s}\left(A_{S}^{T}\right)=\int_{s+s}^{T+s} \alpha_{t}(A) d t, \quad s \in \mathbb{R} .
$$

$$
\frac{\alpha_{s}\left(A_{S}^{T}\right)-A_{S}^{T}}{s}=\frac{1}{s} \int_{S+s}^{s} \alpha_{t}(A) d t+\frac{1}{S} \int_{T}^{T+s} \alpha_{t}(A) d t \underset{s=0}{\longrightarrow} \alpha_{T}(A)-\alpha_{S}(A) .
$$

Proof of Proposition 2. replace $A$ by $A_{S}^{T}$ in (2.10), using (2.11) and (2.12).

\section{Asymptotic Abelianness and Relative Purity}

In addition to dynamical stability as discussed in the last section we now want to impose in essence the asymptotic abelianness of our dynamical system $\{\mathfrak{U}, \alpha\}$ and the relative purity of the stationary state $\omega$. Relative purity in its least specialised version is extremal invariance defined as the fact that $\omega$ cannot be split into a convex combination 
of two different stationary states. If the system $\{\mathfrak{A}, \alpha\}$ is asymptotically abelian this property is known [16-18] to be equivalent to weak clustering defined as the vanishing, in mean, of the second order "truncated expectation value" i.e.

$$
\lim _{T=\infty} \frac{1}{2 T} \int_{-T}^{+T}\left\{\omega\left(A_{1} \alpha_{t}\left(A_{2}\right)\right)-\omega\left(A_{1}\right) \omega\left(A_{2}\right)\right\} d t=0 .
$$

In this work we shall need a technical condition which strengthens (3.1) in that it requires a certain rate of decrease in time, for truncated expectation values up to order 6 . Let us recall that the hierarchy of truncated expectation values $\omega_{n}^{T}, n=1,2, \ldots$, is defined recursively in the following way:

$$
\left\{\begin{array}{l}
0=\omega_{(0)}^{T} \\
\omega\left(A_{1}\right)=\omega_{(1)}^{T}\left(A_{1}\right) \\
\omega\left(A_{1}, A_{2}\right)=\omega_{(2)}^{T}\left(A_{1}, A_{2}\right)+\omega_{(1)}^{T}\left(A_{1}\right) \omega_{(1)}^{T}\left(A_{2}\right) \\
\vdots \\
\omega\left(A_{1}, A_{2}, \ldots A_{n}\right)=\Sigma \omega_{\left(n_{1}\right)}^{T}\left(\prod_{\alpha_{j_{1}}^{1}}^{n_{1}} A_{\alpha_{j_{1}}^{1}}\right) \omega_{\left(n_{2}\right)}^{T}\left(\prod_{\alpha_{j_{2}}^{2}=1}^{n_{2}} A_{\alpha_{j_{2}}^{2}}\right) \\
\quad \ldots \omega_{\left(n_{s}\right)}^{T}\left(\prod_{\alpha_{j_{s}}^{s}=1}^{n_{s}} A_{\alpha_{j_{s}}^{s}}\right)
\end{array}\right.
$$

where the last sum is extended to all partitions

$$
\left\{\alpha_{j_{1}}^{1}\right\}_{j_{1}=1, \ldots n_{1}}\left\{\alpha_{j_{2}}^{2}\right\}_{j_{2}=1, \ldots n_{2}}\left\{\alpha_{j_{s}}^{s}\right\}_{j_{s}=1, \ldots n_{s}}
$$

of the index set $\{1,2, \ldots n\}$ such that $\alpha_{1}^{1}<\alpha_{1}^{2}<\cdots<\alpha_{1}^{n} \alpha_{1}^{k}<\alpha_{2}^{k}<\cdots<\alpha_{n_{k}}^{k}$, $k=1,2, \ldots s$; and $n_{1}+n_{2}+\cdots+n_{s}=n$. We are now able to formulate the "purity condition" which we need.

Definition 3. Let $\omega$ be a stationary state of the dynamical system $(\mathfrak{H}, \alpha\}$. We say that $\omega$ has $L^{1}$-decrease of correlation in time whenever the following prevails: there is a norm dense and self-adjoint subset $\mathscr{S}$ of $\mathfrak{A}$ such that, to any collection $A_{1}, A_{2}, \ldots A_{n}$ of elements of $\mathscr{S}$ with $n \leqq 6$ there are positive constants $C$ and $\delta$ with $^{9}$

$$
\mid \omega_{(n)}^{T}\left(\alpha_{t_{1}}\left(A_{1}\right) \ldots \alpha_{t_{n}}\left(A_{n}\right) \mid \leqq \frac{C}{\left\{1+\operatorname{Sup}_{i, j} \mid t_{i}-t_{j}\right\}^{1+\delta}} .\right.
$$

The next lemma shows that we could have assumed without loss of generality that $\mathscr{S}$ is stable for the operation of smearing out with functions of fast decrease (we will need this fact in the sequel).

${ }^{9}$ The phrase " $\mathscr{S}$ is self adjoint" means that $A^{*} \in \mathscr{S}$ whenever $A \in \mathscr{S}$. It could seem more natural to require a condition of the form (3.3) for all integers $n$. But we need it only for $n \leqq 6$. 
Lemma 3. Let $\mathscr{S} \subseteq \mathfrak{A}$ be such that (3.3) holds with some positive $C$ and $\delta$ for all sets $A_{1}, A_{2}, \ldots A_{n}$ of elements of $\mathscr{S}$. The same holds for the set $\mathscr{S}^{\prime}$ of all $\alpha_{f}(A)$, where $A$ runs through $\mathscr{S}$ and $f$ through the $C^{\infty}$ functions of rapid decrease $\left(\alpha_{f}(A)\right.$ denotes the Bochner integral $\left.\int \alpha_{t}(A) \cdot f(t) d t\right)$.

Proof. We want to infer from inequality (3.3) an inequality of the same type for the expression obtained by replacing there the $A_{i}$ by the $\alpha_{f_{i}}\left(A_{i}\right)$, where the $f_{i}$ are arbitrary $C^{\infty}$-functions with fast decrease. Since these replacements can be performed step by step it is enough that we consider

$$
\begin{aligned}
\omega_{(t)}^{T}\left(\alpha_{t_{1}}\left(A_{1}\right), \ldots, \alpha_{t_{k}}\left(\alpha_{f}\left(A_{k}\right)\right), \ldots, \alpha_{t_{n}}\left(A_{n}\right)\right) \\
\quad=\int \omega_{(n)}^{T}\left(\alpha_{t_{1}}\left(A_{1}\right), \ldots, \alpha_{t_{k}+t}\left(A_{k}\right), \ldots, \alpha_{t_{n}}\left(A_{n}\right)\right) f(t) d t
\end{aligned}
$$

which we want to majorize for each pair of indices $i \neq j$ by an expression of the form $c s t \cdot /\left\{1+\left|t_{i}-t_{j}\right|\right\}^{1+\delta}$. According to (3.3) the truncated expectation value appearing in the above integral is majorized by $C /\left\{1+\left|t_{i}-t_{j}\right|\right\}^{1+\delta}, i \neq k, j \neq k$; as well as by $C /\left\{1+\left|t_{i}-t_{k}-t\right|\right\}^{1+\delta}, i \neq k$. The first majorization, for the case $i \neq k, j \neq k$, yields for the above integral the majorization $C\|f\|_{1} /\left\{1+\left|t_{i}-t_{j}\right|\right\}^{1+\delta}$ of the desired form. The second yields for $j=k \neq i$ the majorization

$$
C \int \frac{|f(t)| d t}{\left\{1+\left|t_{i}-t_{j}-t\right|\right\}^{1+\delta}} .
$$

Assuming $u=t_{i}-t_{j}>0$ we split the integration domain into the intervals $t \notin[0,2 u], 0 \leqq t \leqq u$ and $u \leqq t \leqq 2 u$. The corresponding integrals are respectively majorized by

and

$$
C\|f\| \cdot /\left\{1+\left|t_{i}-t_{j}\right|\right\}^{1+\delta}, \quad C \int_{0}^{u} \frac{|f(t)| d t}{\{1+u-t\}^{1+\delta}}
$$

$$
C \int_{u}^{2 u} \frac{|f(t)| d t}{\{1+t-u\}^{1+\delta}}
$$

the first expression being already of the desired type, whilst the two others are easily shown to be majorized by an expression of the type $\operatorname{cst} \cdot /\{1+u\}^{1+\delta}$ using an appropriate majorization of $f$. The case $u<0$ is treated analogously.

As is to be expected, Definition 3 implies strong clustering of $\omega$. Specifically

Lemma 4. If the stationary state of the dynamical system $\{\mathfrak{U}, \alpha\}$ has $\left(L^{(1)}\right.$-decrease of correlations in time the functions $F_{A_{2} A_{1}}, G_{A_{1} A_{2}}$, and 
$H_{A_{1} A_{2} A_{3}}, A_{1}, A_{2}, A_{3} \in \mathfrak{U}$, defined by

$$
\begin{aligned}
F_{A_{2} A_{1}}(t) & =\omega\left(A_{1} \alpha_{t}\left(A_{2}\right)\right)-\omega\left(A_{1}\right) \omega\left(A_{2}\right) \\
G_{A_{1} A_{2}}(t) & =\omega\left(\alpha_{t}\left(A_{1}\right) A_{2}\right)-\omega\left(A_{1}\right) \omega\left(A_{2}\right) \\
H_{A_{1} A_{2} A_{3}}(t) & =\omega\left(A_{1} \alpha_{t}\left(A_{2}\right) A_{3}\right)-\omega\left(A_{1} A_{3}\right) \omega\left(A_{2}\right)
\end{aligned}
$$

all belong to $L^{1}(R)$ for $A_{1}, A_{2}, A_{3} \in \mathscr{S}$. These functions vanish at $\infty$ if $A_{1}, A_{2}, A_{3} \in \mathfrak{U}$. As a consequence $\pi_{\omega}\left(\alpha_{t}(A)\right)$ tends $\sigma$-weakly towards $\omega(A) I$ for all $A \in \mathfrak{A}\left(\pi_{\omega}\right.$ is the representation of $\mathfrak{A}$ generated by $\left.\omega\right)$.

Proof. The first part of the lemma follows from (3.3) noticing that

$$
\begin{aligned}
F_{A_{2} A_{1}}(t) & =\omega_{(2)}^{T}\left(A_{1} \alpha_{t}\left(A_{2}\right)\right) \\
G_{A_{1} A_{2}}(t) & =\omega_{(2)}^{T}\left(\alpha_{t}\left(A_{1}\right) A_{2}\right) \\
H_{A_{1} A_{2} A_{3}}(t) & =\omega_{(3)}^{T}\left(A_{1} \alpha_{t}\left(A_{2}\right) A_{3}\right)+\omega\left(A_{3}\right) \omega_{(2)}^{T}\left(A_{1} \alpha_{t}\left(A_{2}\right)\right) \\
& +\omega\left(A_{1}\right) \omega_{(2)}^{T}\left(\alpha\left(A_{2}\right) A_{3}\right) .
\end{aligned}
$$

The rest follows using sequences $\left\{A_{1}^{n}\right\},\left\{A_{2}^{n}\right\},\left\{A_{3}^{n}\right\}$ in $\mathscr{S}$ converging in norm respectively towards $A_{1}, A_{2}, A_{3} \in \mathfrak{A}$.

We now prove a technical result for use in the proof of Section 4. This result is included in the present section because it depends merely on Definition 3 above ${ }^{10}$.

Lemma 5. Assume that the stationary state $\omega$ of the dynamical system $\{\mathfrak{A}, \alpha\}$ has $L^{1}$-decrease of correlation in time; let $A_{1}, A_{2}, A_{3}, h_{1}, h_{2}, h_{3}$ be elements of $\mathscr{S}$; and consider the functions

and

$$
\begin{aligned}
\Phi_{u}(t)= & \omega\left(\left[h_{1} \alpha_{u}\left(h_{2}\right), \alpha_{t}\left(A_{1} \alpha_{u}\left(A_{2}\right)\right)\right]\right) \\
& -F_{A_{1} h_{1}}(t) F_{A_{2} h_{2}}(t)+G_{A_{1} h_{1}}(t) G_{A_{2} h_{2}}(t)
\end{aligned}
$$

$$
\begin{aligned}
\psi_{u}(t)= & \omega\left(\left[h_{1} \alpha_{u}\left(h_{2}\right) \alpha_{2 u}\left(h_{3}\right), \alpha_{t}\left(A_{1} \alpha_{u}\left(A_{2}\right) \alpha_{2 u}\left(A_{3}\right)\right)\right]\right) \\
& -F_{A_{1} h_{1}}(t) F_{A_{2} h_{2}}(t) F_{A_{3} h_{3}}(t)+G_{A_{1} h_{1}}(t) G_{A_{2} h_{2}}(t) G_{A_{2} h_{3}}(t)
\end{aligned}
$$

where $u$ is a real parameter. One has $\phi_{u}=\phi_{u}^{(1)}+\phi_{u}^{(2)}$ and $\psi_{u}=\psi_{u}^{(1)}+\psi_{u}^{(2)}$ where

(i) $\phi_{u}^{(1)}$ and $\psi_{u}^{(1)}$ are majorized in module by an $L^{1}$-function independent of $u$ and tend point wise to zero for $u \rightarrow \infty$,

(ii) $\phi_{u}^{(2)}$ is a linear combination of functions of the type $F_{A h}-G_{A h}$ with $A, h \in \mathscr{S}$,

(iii) $\psi_{u}^{(2)}$ is a linear combination of functions of the type considered in (ii) and functions of the type $F_{A h} F_{A^{\prime} h^{\prime}}-G_{A h} G_{A^{\prime} h^{\prime}}, A, A^{\prime}, h, h^{\prime} \in \mathscr{S}^{11}$.

10 The reader might prefer to skip the end of this section and return to it as the results are needed in the proof of the Section IV.

11 In (ii) and (iii) the coefficients of the linear combinations, as well as the elements $A, A^{\prime}, h, h^{\prime}$ are allowed to depend upon the parameter $u$. 
Proof. The proof consists in a straightforward if somewhat cumbersome verification: one expands according to (3.2) the first terms of the r.h.s. of (3.5) and (3.6) in terms of truncated expectation values. Examination of the latter then yields the result, taking account of the majorizations (3.3). We first note that in these majorizations the time differences $\left|t_{i}-t_{j}\right|$ which appear are $|u|,|t|,|t+u|,|t-u|,|t+2 u|,|t-2 u|$. Whenever at least two of the latter are present, say a and b, we have due to (3.3) for the corresponding term a majorization of the type Cste $/(1+a)^{\alpha}(1+b)^{\beta}$, where $\alpha$ and $\beta$ are any positive constants with $\alpha+\beta \leqq 1+\delta$. From this it follows immediately that for all choices of $a$ and $b$ amongst the timedifferences listed above, we obtain a majorization of the type $f(u) /(1+|t|)^{1+\varepsilon}$, with $\varepsilon>0$ and $f$ continuous bounded tending to zero pointwise for $u \rightarrow \infty$; therefore the corresponding term then yields a contribution to $\phi_{u}^{(1)}$ or $\psi_{u}^{(1)}$ as described under (i) in the lemma.

With this in mind let us now examine the expansion (3.2) for the first r.h.s. term in (3.5), going through the different partitions of the set $\left\{h_{1} h_{2} A_{1} A_{2}\right\}$. Amongst these only the ones containing "heterogeneous subsets" (i.e. subsets containing at last one $A$ and one $h$ ) will yield non vanishing contributions, since the others give expressions cancelling out for the two terms of the commutator. Thus the partitions to be considered, classified by the cardinals of their subsets, fall into the four types: (4); (3)(1); (2)(1)(1); (2) (2) with the restriction that at least one of their subsets has to be heterogeneous. Now the two first types will necessarily give rise to at least two of the time differences considered above, and therefore yield contributions to $\phi_{u}^{(1)}$. The third type on the other hand yields contributions to $\phi_{u}^{(2)}$. Finally there are two partitions of the type (2) (2): $\left(h_{1} A_{2}\right)\left(h_{2} A_{1}\right)$ which gives rise to two of the above time-differences and thus contributes a term to $\phi_{u}^{(1)}$; and $\left(h_{1} A_{1}\right)\left(h_{2} A_{2}\right)$ which yields the second term in the r.h.s. of (3.5).

For $\psi_{u}$ the proof goes along similar lines. All partitions containing a subset of at least three elements gives rise to at least two time-differences, thus yielding a contribution to $\psi_{u}^{(1)}$. The remaining partitions fall into the three types (2)(1)(1)(1)(1); (2)(2)(1)(1); or (2)(2)(2). The first type yields terms of the form $F_{A h}-G_{A h}$; the second, terms of this type or of the type $F_{A h} F_{A^{\prime} h^{\prime}}-G_{A h} G_{A^{\prime} h^{\prime}}$ according to wether it contains one or two heterogeneous subsets); the last type finally either contains only one heterogeneous subset, in which case it gives rise to terms in $\phi_{u}^{(1)}$; or is the partition $\left(h_{1} A_{1}\right)\left(h_{2} A_{2}\right)\left(h_{3} A_{3}\right)$, which contributes the second term in the r.h.s. of (3.6).

We end this section with a discussion of the implications of asymptotic abelianness and relative purity on the spectrum of the energy. We do not claim originality for the proposition to follow, which is merely a reminder of a situation known to physicists since a long time (see, e.g., $[19,10]$ ), 
and which appears in several variants and in a modernized form in the recent mathematical literature [21]-[24]. Let us first recall a few definitions relating to a dynamical system $\{\mathfrak{U}, \alpha\}$ or to a continuous unitary representation $U$ of the reals on a Hilbert space $\mathscr{H}$. With $f \in L^{1}(\mathbb{R}), A \in \mathfrak{U}$ and $\psi \in \mathscr{H}$, we write

$$
\begin{aligned}
\alpha_{f}(A) & =\int f(t) \alpha_{t}(A) d t \\
U_{f} \psi & =\int f(t) U_{t} \psi d t
\end{aligned}
$$

(the integrals are in the Bochner sense). We then define the subsets $\operatorname{Sp}(\alpha), \operatorname{Sp}(U), \operatorname{Sp}_{\alpha}(A)$ and $\operatorname{Sp}_{U}(\psi)$ of the dual real line $\hat{\mathbb{R}}$ by the following equivalences

$$
\begin{array}{rlllr}
E \in \operatorname{Sp}(\alpha) \Leftrightarrow \hat{f}(E)=0 & \text { for all } & f \in L^{1}(\mathbb{R}) & \text { with } & \alpha_{f}=0 \\
E \in \operatorname{Sp}(U) \Leftrightarrow \hat{f}(E)=0 & \text { for all } & f \in L^{1}(\mathbb{R}) & \text { with } & U_{f}=0 \\
E \in \operatorname{Sp}_{\alpha}(A) \Leftrightarrow \hat{f}(E)=0 & \text { for all } & f \in L^{1}(\mathbb{R}) & \text { with } & \alpha_{f}(A)=0 \\
E \in \operatorname{Sp}_{U}(\psi) \Leftrightarrow \hat{f}(E)=0 & \text { for all } & f \in L^{1}(\mathbb{R}) & \text { with } & U_{f} \psi=0
\end{array}
$$

where $\hat{f}$ denotes the Fourier transform of $f$. We recall the following properties [23]: these four subsets defined by (3.7) are closed; $\operatorname{Sp}_{\alpha}\left(\alpha_{t}(A)\right)=\operatorname{Sp}_{\alpha}(A)$ for all $t \in \mathbb{R} ; \operatorname{Sp}_{\alpha}\left(A_{1} A_{2}\right) \subseteq \operatorname{Sp}_{\alpha}\left(A_{1}\right)+\operatorname{Sp}_{\alpha}\left(A_{2}\right)$ for all $A_{1}, A_{2} \in \mathfrak{A} ; \operatorname{Sp}_{\alpha}\left(\alpha_{f}(B)\right) \subseteq \operatorname{Sp}_{\alpha}(B) \cap$ support $\hat{f}$ for all $B \in \mathfrak{A}$ and $f \in L^{1}(R)$; finally if $P_{U}$ denotes the spectral measure of the representation $U$ we have that $P_{U}(\Delta) \mathscr{H}=\left\{\psi \in \mathscr{H} \mid \mathrm{Sp}_{U}(\psi) \cong \Delta\right\}$ for each closed subset $\Delta$ of $\hat{R}$. We can now state.

Proposition 3. Let $(\pi, U)$ be the covariant representation of the dynamical system $\{\mathfrak{A}, \alpha\}$ generated by the stationary state $\omega$ and assume $\omega$ to be such that the function $H_{A_{1} A_{2} A_{3}}$ defined in Lemma 4 vanishes for $t=\infty$ for all $A_{1}, A_{2}, A_{3} \in \mathfrak{A}$. The spectrum of $U$ is then additive. Furthermore this spectrum is either one-sided, or covers the whole real line (i.e. either $\mathrm{Sp}(U) \subseteq \hat{R}^{+}$, or $\mathrm{Sp}(U) \subseteq \hat{R}^{-}$, or $\left.\mathrm{Sp}(U)=\hat{R}\right)$.

For the proof we need the following ${ }^{12}$.

Lemma 6. Let $(\pi, U)$ be the covariant representation of $\{\mathfrak{U}, \alpha\}$-with cyclic invariant vector $\Omega$, generated by the stationary state $\omega$; and assume $\mathscr{S}$ to be a norm-dense subset of $\mathfrak{U}$ stable for all $\alpha_{f}, f \in \mathscr{F}^{-1}(\mathscr{D})$. The following are equivalent for $E \in \hat{R}$

(i) $E \in \operatorname{Sp}(U)$.

(ii) to each neighbourhood $\mathscr{V}$ of $E$ there is an $A \in \mathscr{S}$ with $\pi(A) \Omega \neq 0$ and $\operatorname{Sp}_{\alpha}(A) \subseteq \mathscr{V}$.

Proof. (i) $\Rightarrow$ (ii): Let $E \in \mathrm{Sp}(U)$ with $\mathscr{V}$ any neighbourhood of $E$. Let us choose $f \in \mathscr{F}^{-1}(\mathscr{D})$, so that $\hat{f} \in \mathscr{D}$, with the support of $\hat{f}$ within

${ }_{12}$ In fact the only case needed to prove Proposition 3 is that of $\mathscr{S}=\mathfrak{A}$. We formulate and prove Lemma 6 as it stands because we will need it in that form for the argument in Section IV. 
$\mathscr{V}$ and $\hat{f}(E)=1$. Since $E \in \operatorname{Sp}(U)$ one has $U_{f} \neq 0$, thus by the density of $\mathscr{S}$ in $\mathfrak{A}$ and the cyclicity of $\Omega$, there is $B \in \mathscr{S}$ with $U_{f} \pi(B) \Omega$ $=\pi\left(\alpha_{f}(B)\right) \Omega \neq 0$; therefore $A=\alpha_{f}(B)$ is such that $\pi(A) \Omega \neq 0$ and, since $\operatorname{Sp}_{\alpha}\left(\alpha_{f}(B)\right) \cong$ Support $\hat{f}$, one has $\operatorname{Sp}_{\alpha}(A) \cong \mathscr{V}$.

(ii) $\Rightarrow$ (i): Since $\operatorname{Sp}_{U}(\pi(A) \Omega) \cong \operatorname{Sp}_{\alpha}(A)$ due to $\pi\left(\alpha_{f}(A) \Omega\right)=U_{f} \pi(A) \Omega$ for all $f \in L^{1}(R)$ we see that (ii) implies $P_{U}(\mathscr{V}) \neq 0$ for each closed neighbourhood $\mathscr{V}$ of $E$. Thus $E \in \operatorname{Sp}(U)$.

Proof of Proposition 3. Let $E_{1}, E_{2} \in \mathrm{Sp}(U)$ we want to show that $E_{1}+E_{2} \in \mathrm{Sp}(U)$. By the preceding lemma this holds if, given any neighbourhood $\mathscr{V}$ of $E_{1}+E_{2}$, there is $A \in \mathfrak{A}$ with $\operatorname{Sp}_{\alpha}(A) \in \mathscr{V}$ and $\pi(A) \Omega \neq 0$. To construct such an $A$, select first respective neighbourhoods $\mathscr{V}_{1}, \mathscr{V}_{2}$ of $E_{1}, E_{2}$ with $\mathscr{V}_{1}+\mathscr{V}_{2} \subseteq \mathscr{V}$. Again by Lemma 6 we can find $A_{1}, A_{2} \in \mathfrak{O}$ with $\pi\left(A_{1}\right) \Omega \neq 0, \pi\left(A_{2}\right) \Omega \neq 0, \mathrm{Sp}_{\alpha}\left(A_{1}\right) \leqq \mathscr{V}_{1}, \mathrm{Sp}_{\alpha}\left(A_{2}\right) \leqq \mathscr{V}_{2}$. Let $t \in R$, we have

$$
\left\|\pi \alpha_{t}\left(A_{1}\right) A_{2} \Omega\right\|^{2}=\omega\left(A_{2}^{*} \alpha_{t}\left(A_{1}^{*} A_{1}\right) A_{2}\right)
$$

which by assumption tends towards $\omega\left(A_{2}^{*} A_{2}\right) \omega\left(A_{1}^{*} A_{1}\right) \neq 0$ for $t=\infty$. Therefore $t$ can be chosen so that $\pi\left(\alpha_{t}\left(A_{1}\right) A_{2}\right) \Omega \neq 0$. Since on the other hand $\operatorname{Sp}_{\alpha}\left(\alpha_{t}\left(A_{1}\right) A_{2}\right) \cong \operatorname{Sp}_{\alpha}\left(\alpha_{t}\left(A_{1}\right)\right)+\operatorname{Sp}_{\alpha}\left(A_{2}\right)$ we have for $A=\alpha_{t}\left(A_{1}\right) A_{2}$ the desired properties $\pi(A) \Omega \neq 0$ and $\operatorname{Sp}_{\alpha}(A) \cong \mathscr{V}$.

Now we want to show that if $\operatorname{Sp}(U)$ is not one-sided it coincides with the whole real line. Assume thus that $-a$ and $b$ belong to $\operatorname{Sp}(U)$ with $a>0$ and $b>0$. Then $m b-n a \in \operatorname{Sp}(U)$ for all positive integers $m$ and $n$ by the additivity of $\operatorname{Sp}(U)$. If $a$ and $b$ have no common divisor 0 is an accumulation point of the set $\{m b-n a, m, n$ positive integers $\}$, thus $\operatorname{Sp}(U)$ has elements of arbitrarily small module. Adding appropriate multiples of these to either $b$ or $-a$ we can construct elements of $\operatorname{Sp}(U)$ arbitrarily close to 0 on both sides, whose multiples yield in turn points in $\operatorname{Sp}(U)$ arbitrarily close to any preassigned real number. Thus $\operatorname{Sp}(U)$ covers the whole real since it is closed. In the case where $a$ and $b$ have a common divisor, i.e. when $m b-n a=0$ for some positive integers $m$ and $n$ we argue as follows. We note that the assumed vanishing of $H_{A_{1} A_{2} A_{3}}$ for arbitrary $A_{1}, A_{2}, A_{3} \in \mathscr{S}$ excludes the existence of an isolated point $E_{0} \neq 0$ in $\operatorname{Sp}(U)$, since Lemma 6 would in this case yield an $A \in \mathfrak{A}$ with $\alpha_{t}(A)=e^{i E_{0} t} A$ for all $t \in R$. Consequently there must be an element of $\operatorname{Sp}(U)$ arbitrarily close to $-a$, say $a^{\prime}$, which would furnish points $m b-n a^{\prime} \in \operatorname{Sp}(U)$ of arbitrarily small module, allowing us to proceed as above.

\section{Derivation of the KMS Condition}

We now draw joint consequences of assumptions (i), (ii), (iii) of the Introduction as made precise in the two preceding sections. Our aim 
is the theorem below for the proof of which the two following propositions are key devices.

Proposition 4. Assume the stationary state $\omega$ of the dynamical system $\{\mathfrak{U}, \alpha\}$ to be folium-stable for inner perturbations and to have $L^{1}$-decrease of correlations in time. One has, for all $A, h \in \mathfrak{U}$

$$
\int_{-\infty}^{+\infty} \omega\left(\left[h, \alpha_{t}(A)\right]\right) d t\left(=\int_{-\infty}^{+\infty}\left[F_{A h}(t)-G_{A h}(t)\right] d t\right)=0
$$

with independent limits of integration.

Proof. By linearity, it suffices to prove the result for $h$ self-adjoint. This is achieved by passing to the limits $S \rightarrow-\infty, T \rightarrow+\infty$ in (2.9) above, whose left hand side then vanishes due to the fact that $\pi_{\omega}\left(\alpha_{t}(A)\right) \underset{t= \pm \infty}{\longrightarrow} \omega(A) I \quad \sigma$-weakly (see Lemma 4) whilst the linear form $\omega_{1}^{(h)}$, normal for the representation $\pi_{\omega}$, vanishes on multiple of the unit.

Proposition 5. With the same assumptions as in Proposition 4 one has for all $A_{1}, A_{2}, A_{3}, h_{1}, h_{2}, h_{3} \in \mathscr{S}$

and

$$
\int F_{A_{1} h_{1}}(t) F_{A_{2} h_{2}}(t) d t=\int G_{A_{1} h_{1}}(t) G_{A_{2} h_{2}}(t) d t
$$

$$
\int F_{A_{1} h_{1}}(t) F_{A_{2} h_{2}}(t) F_{A_{3} h_{3}}(t) d t=\int G_{A_{1} h_{1}}(t) G_{A_{2} h_{2}}(t) G_{A_{3} h_{3}}(t) d t
$$

where $F_{A h}$ is defined as in (3.4).

Proof. Integrate both sides of Eqs. (3.5) and (3.6): the first terms on the right hand sides will yield zero due to (4.1). And the left hand sides yield integrals which vanish in the limits $u \rightarrow \infty$, resp $u$, and $v \rightarrow \infty$, due to Lemma 5; plus terms vanishing due to (4.1) or (4.2).

From now on we suppose, in addition to the assumptions of Proposition 4, that the energy spectrum in the representation generated by $\omega$ covers the whole real line. We show as our next step that, for $A, h \in \mathscr{S}$, the Fourier transforms $\hat{F}_{A h}$ and $\hat{G}_{A h}$ of $F_{A h}$, resp $G_{A h}$ differ by a factor which is a function of energy, independent of the choice of $A$ and $h$. From Lemma 4 we know that these Fourier transforms are continuous functions. On the other hand (4.2) yields

$$
\int \hat{F}_{A_{1} h_{1}}(E) \hat{F}_{A_{2} h_{2}}(-E) d E=\int \hat{G}_{A_{1} h_{1}}(E) \hat{G}_{A_{2} h_{2}}(-E) d E
$$

for all $A_{1}, A_{2}, h_{1}, h_{2} \in \mathscr{S}$. Now fix $E_{0} \in R, E_{0}$ belongs to the spectrum of the energy, therefore we can, by Lemma 6 , choose to each positive integer $n$ an element $A_{(n)} \neq 0$ of $\mathscr{S}$ with $S p_{\alpha}\left(A_{(n)}\right) \subseteq\left[E_{0}-\frac{1}{n}, E_{0}+\frac{1}{n}\right]$. 
Choosing $h_{(n)}=\lambda_{n} A_{(n)}^{*}$ with $\lambda_{n}$ positive, the functions $\hat{F}_{A_{(n)} h_{(n)}}$, $\hat{G}_{A_{(n)} h_{(n)}}$ are positive, have support in $\left[E_{0}-\frac{1}{n}, E_{0}+\frac{1}{n}\right]$ and, by suitable choice of $\lambda_{n}$ we may normalize $\hat{F}$ so that

$$
\int \hat{F}_{A_{(n)} h_{(n)}}(-E) d E=1 \text {. }
$$

Taking now in (4.4) $A_{1}=A, h_{1}=h, A_{2}=A_{(n)}, h_{2}=h_{(n)}$ the left hand side converges for $n \rightarrow \infty$ and we get

$$
\hat{F}_{A h}\left(E_{0}\right)=\hat{G}_{A h}\left(E_{0}\right) \cdot \lim _{n \rightarrow \infty} \int \hat{G}_{A_{(n)} h_{(n)}}(-E) d E .
$$

Since the sequence $A_{(n)}, h_{(n)}$ was constructed in a manner depending only on $E_{0}$ and not on $A$ or $h$ this shows the existence of a function $\phi(E)$ such that

$$
\hat{F}_{A h}(E)=\phi(E) \hat{G}_{A h}(E)
$$

for all $A, h \in \mathscr{S}$.

Now it is immediate from the definition of $F_{A h}$ and $G_{A h}$ that one has

$$
\bar{F}_{h^{*} A^{*}}(t)=F_{A h}(-t)=G_{h A}(t)
$$

whence the facts that $\hat{G}_{A^{*} A}$ and $\hat{F}_{A^{*} A}$ are real, $A \in \mathfrak{Q}$, and

$$
\hat{F}_{A h}(-E)=\hat{G}_{h A}(E), \quad E \in \hat{R}, \quad A, h \in \mathfrak{A} .
$$

Comparison with (4.5) shows that $\phi$ is real and satisfies

$$
\phi(-E)=\phi(E)^{-1}, \quad E \in \hat{R}
$$

Finally, inserting (4.5) in

$$
\begin{aligned}
\int \hat{F}_{A_{1} h_{1}}(E) \hat{F}_{A_{2} h_{2}}\left(E^{\prime}-E\right) \hat{F}_{A_{3} h_{3}}\left(-E^{\prime}\right) d E d E^{\prime} \\
=\int \hat{G}_{A_{1} h_{1}}(E) \hat{G}_{A_{2} h_{2}}\left(E^{\prime}-E\right) \hat{G}_{A_{3} h_{3}}\left(-E^{\prime}\right) d E d E^{\prime}
\end{aligned}
$$

obtained from (4.3) we see that

$$
\int\left[1-\phi(E) \phi\left(E^{\prime}-E\right) \phi\left(-E^{\prime}\right)\right] \hat{G}_{A_{1} h_{1}}(E) \hat{G}_{A_{2} h_{2}}\left(E^{\prime}-E\right) \hat{G}_{A_{3} h_{3}}(-E) d E d E^{\prime}=0 \text {. }
$$

Arguing as above with sequences $A_{1_{(n)}}$ and $A_{3_{(n)}}$ spectrally concentrated around $E_{0}$ and $E_{0}^{\prime}$ respectively we conclude from this that

$$
\phi\left(E_{0}\right) \phi\left(E_{0}^{\prime}-E\right) \phi\left(-E_{0}^{\prime}\right)=1, \quad E_{0}, E_{0}^{\prime} \in \hat{R}
$$

and thus, using (4.8), that the function $\phi$ is multiplicative: we have therefore that $\phi(E)=e^{\beta E}$ for some real constant $\beta$. We have reached the KMS condition [11]

$$
\hat{F}_{A h}(E)=e^{\beta E} \hat{G}_{A h}(E)
$$

for $A, h \in \mathscr{S}$. There remains to show that (4.9) is true for arbitrary $A, h \in \mathfrak{A}$. To this end we notice that, in the general case, $F_{A h}$ and $\hat{G}_{A h}$ 
are bounded measures such that

$$
\begin{aligned}
& d \hat{F}_{A h}(E)=\left(h^{*} \Omega\left|d P_{U}(E)\right| A \Omega\right) \\
& d \hat{G}_{A h}(E)=\left(A^{*} \Omega\left|d P_{U}(E)\right| h \Omega\right)
\end{aligned}
$$

where $P_{U}$ is the spectral measure of the representation $U$. Thus the condition replacing (4.9) for general $A, h \in \mathfrak{A}$, namely

$$
\hat{F}_{A B}=\phi \cdot \hat{G}_{A h} \quad \text { with } \quad \phi(E)=e^{\beta E},
$$

can be expressed as the fact that

$$
\left(h^{*} \Omega|f(H)| A \Omega\right)=\left(A^{*} \Omega\left|e^{\beta H} f(H)\right| h \Omega\right)
$$

for all $f(H)$, where $H$ is the hamiltonian operator (defined by $e^{i H t}=U_{t}$, $t \in R$ ) and $f$ any continuous function with compact support. Under the latter form it is evident that the KMS condition (4.11) need only be checked for $A, h$ in the dense set $\mathscr{S}$.

Conferring what we have shown with the alternative in Proposition 3 we obtain the

Theorem. Let $\omega$ be a state of the dynamical system $\{\mathfrak{U}, \alpha\}$ which:

(i) is stationary;

(ii) is folium-stable for local (or inner) perturbations of the dynamics;

(iii) has $L^{1}$-decrease of correlations in time.

Then either the spectrum of the energy is one-sided in the representation generated by $\omega$, or $\omega$ is a KMS state.

\section{Appendix - The Ideal Fermi Gas}

This example provides a simple illustration of the determination of equilibrium states by the stability condition (1.11) or (4.1). We give only a brief sketch of the argument. For a more complete discussion see [8]. Here $\mathfrak{A}$ is the gauge invariant part of the CAR-algebra $\mathscr{F}^{13}$. Writing symbolically $a^{*}(f)=\int f(\boldsymbol{p}) a^{*}(\boldsymbol{p}) d^{3} p, a(f)=\int \bar{f}(\boldsymbol{p}) a(\boldsymbol{p}) d^{3} p$ the time translation automorphism for a medium composed of noninter-

$13 \mathscr{F}$ is the $C^{*}$-algebra generated by particle annihilators $a(f)$ satisfying the canonical anticommutation relations

$$
\begin{gathered}
{\left[a(f), a^{*}(g)\right]_{+}=a(f) a^{*}(g)+a^{*}(g) a(f)=(f, g) ; \quad[a(f), a(g)]_{+}=0 ;} \\
f, g \in \mathscr{L}^{(2)}\left(\mathbb{R}^{3}\right) .
\end{gathered}
$$

$\mathfrak{U}$ is the subalgebra of the invariant elements under the "gauge transformation

$$
a(f) \rightarrow e^{\imath \phi} a(f) \quad(\phi \text { a real number }) .
$$


acting, identical Fermi particles can be characterized by

$$
\alpha_{t}\left(a^{*}(\boldsymbol{p})\right)=e^{i \varepsilon(\boldsymbol{p}) t} a^{*}(\boldsymbol{p}),
$$

where $\varepsilon(p)$ is the energy of a single particle with momentum $p$.

We show first that (1.11) (together with the required stationarity and relative purity) implies that the state $\omega$ is quasi-free and homogeneous, i.e. $\omega$ is completely describable in terms of a single-particle momentum distribution $\varrho(p)$ by

$$
\begin{aligned}
\omega\left(\alpha^{*}\left(f_{1}\right) \ldots a^{*}\left(f_{n}\right) a\left(f_{n}^{\prime}\right) \ldots a\left(f_{1}^{\prime}\right)\right) & =\operatorname{det} \alpha_{i k} \\
\alpha_{i_{k}} & =\int \bar{f}_{i}^{\prime}(\boldsymbol{p}) \varrho(\boldsymbol{p}) f_{k}(\boldsymbol{p}) d^{3} p .
\end{aligned}
$$

To see this let us choose in (1.11)

$$
A=a^{*}\left(f_{1}\right) \ldots a^{*}\left(f_{n}\right) a\left(f_{n}^{\prime}\right) \ldots a\left(f_{1}^{\prime}\right) ; \quad h=a^{*}(g) a\left(g^{\prime}\right)
$$

with $f_{i}, f_{i}^{\prime}, g, g^{\prime}$ test functions of class $\mathscr{D}$ whose supports we denote respectively by $K_{i}, K_{i}^{\prime}, K, K^{\prime}$. If $K$ is disjoint from $K_{2}^{\prime} \cup K_{3}^{\prime} \cup \cdots \cup K_{n}^{\prime}$ and $K^{\prime}$ disjoint from $K_{2} \cup K_{3} \cup \cdots \cup K_{n}$ then (1.11) gives

where

$$
\begin{aligned}
& \omega\left(a^{*}\left(f_{1}\right) \ldots a^{*}\left(f_{n}\right) a\left(f_{n}^{\prime}\right) \ldots a\left(f_{2}^{\prime}\right) a\left(F^{\prime}\right)\right) \\
& =\omega\left(a^{*}(F) a^{*}\left(f_{2}\right) \ldots a^{*}\left(f_{n}\right) a\left(f_{n}^{\prime}\right) \cdot a\left(f_{1}^{\prime}\right)\right)
\end{aligned}
$$

$$
\begin{aligned}
& F(\boldsymbol{q})=g(\boldsymbol{q}) \int \delta\left(\varepsilon(\boldsymbol{q})-\varepsilon\left(\boldsymbol{q}^{\prime}\right)\right) \bar{g}\left(\boldsymbol{q}^{\prime}\right) f_{1}\left(\boldsymbol{q}^{\prime}\right) d^{3} q^{\prime} \\
& F^{\prime}(\boldsymbol{q})=g^{\prime}(\boldsymbol{q}) \int \delta\left(\varepsilon(\boldsymbol{q})-\varepsilon\left(\boldsymbol{q}^{\prime}\right)\right) \bar{g}\left(q^{\prime}\right) f_{1}^{\prime}\left(q^{\prime}\right) d^{3} q^{\prime} .
\end{aligned}
$$

Suppose we are given test functions $f_{2}, \ldots f_{n}, f_{1}^{\prime} \ldots f_{n}^{\prime}$ and a compact $\hat{K}_{1} \subset \mathbb{R}^{3}$, disjoint from the origin and disjoint from $\cup K_{i}^{\prime}(i=1, \ldots n)$, then, if the supports $K_{i}$ are small enough ${ }^{14}$ we can find functions $\hat{g}$, $f_{1} \in \mathscr{D}$ with supports disjoint from $K_{2}, \ldots K_{n}$ so that

satisfies

$$
\begin{gathered}
\phi(\boldsymbol{q})=\int \delta\left(\varepsilon(\boldsymbol{q})-\varepsilon\left(\boldsymbol{q}^{\prime}\right)\right) \bar{g}^{\prime}\left(\boldsymbol{q}^{\prime}\right) f_{1}\left(\boldsymbol{q}^{\prime}\right) d^{3} q^{\prime} \\
\inf _{\boldsymbol{q} \in \hat{K}_{1}}|\phi(\boldsymbol{q})|>0
\end{gathered}
$$

and $\phi^{-1}$ is infinetely differentiable within $\hat{K}_{1}$.

In this situation, we can then represent an arbitrary $F \in \mathscr{D}\left(\hat{K}_{1}\right)$ by (A.7) with $g \in \mathscr{D}\left(\hat{K}_{1}\right)$ giving us $F^{\prime}=0$ since $\hat{K}_{1}$ and $K_{1}^{\prime}$ are disjoint. This means that the expectation value on the right hand side of (A.6) has to vanish whenever the support of $F$ is disjoint from the origin and

${ }^{14}$ It will suffice to require $S_{R} \cap\left(K_{2} \cup \cdots \cup K_{n}\right)_{c} \neq \emptyset$ for

$$
\inf _{\boldsymbol{q} \in \hat{K}_{1}}\left|\boldsymbol{q} \leqq R \leqq \operatorname{Sup}_{\boldsymbol{q} \in \hat{\mathrm{K}}_{1}}\right| \boldsymbol{q} \mid
$$

$\left(S_{R}\right.$ denotes the surface of a sphere with radius $R$ around the origin, the index $c$ the complement.) 
from all the supports of $f_{i}^{\prime}(i=1, \ldots n)$ and if the supports of $f_{i}(i=2, \ldots n)$ are small enough. In other words, the distribution

$$
W^{(2 n)}\left(\boldsymbol{p}_{1} \ldots \boldsymbol{p}_{n} ; \boldsymbol{p}_{n}^{\prime} \ldots \boldsymbol{p}_{1}^{\prime}\right)=\omega\left(a^{*}\left(p_{1}\right) \ldots a^{*}\left(p_{n}\right) a\left(p_{n}^{\prime}\right) \ldots a\left(p_{1}^{\prime}\right)\right)
$$

has point support at the origin in at least one of the variables $\left(p_{1}-p_{i}^{\prime}\right)$ $(i=1, \ldots n)$ or $p_{1}$.

"Since the expressions (A.6) should be bounded by the $\mathscr{L}^{(2)}$-norms of the test function the only allowed possibility is $\delta\left(\boldsymbol{p}_{1}-\boldsymbol{p}_{i}\right)$. Since the index 1 plays no special role we can repeat the argument, replacing $\boldsymbol{p}_{1}$ by any $p_{i}$ and conclude that $W^{(2 n)}$ must contain a product of $n$ factors $\delta\left(\boldsymbol{p}_{i}-\boldsymbol{p}_{j}^{\prime}\right)$. This property will persist in the truncated distributions. For $n>1$ this implies that unless $W_{T}^{(2 n)}=0$ the correlation functions (3.3) will not for all $i, j$ decrease as they should as $\left(t_{i}-t_{j}\right) \rightarrow \infty$. We conclude

and

$$
W_{T}^{(2 n)}=0 \quad \text { for } \quad n>1
$$

$$
W^{(2)}\left(\boldsymbol{p}, \boldsymbol{p}^{\prime}\right)=\varrho(\boldsymbol{p}) \delta\left(\boldsymbol{p}-\boldsymbol{p}^{\prime}\right)
$$

with $\varrho$ a positive measure. In other words $\omega$ has to be of the form (A.4).

It is clear that for arbitrary $\varrho$ the state (A.4) is stationary and relatively pure. Using the stability condition (1.11) once more we show that $\varrho$ has to be the Fermi distribution. For this purpose we choose $A$ as in (A.5) with $n=2$ but $h=A^{*}$. Entering with this into (1.11), choosing $K_{1}$ disjoint from $K_{2}^{\prime}, K_{2}$ disjoint from $K_{1}^{\prime}$ we get

$$
\begin{aligned}
& \int\left|f_{1}\left(\boldsymbol{p}_{1}\right)\right|^{2}\left|f_{2}\left(p_{2}\right)\right|^{2}\left|f_{2}^{\prime}\left(\boldsymbol{p}_{2}^{\prime}\right)\right|^{2}\left|f_{1}^{\prime}\left(\boldsymbol{p}_{1}^{\prime}\right)\right|^{2} \delta\left(\varepsilon\left(\boldsymbol{p}_{1}\right)+\varepsilon\left(\boldsymbol{p}_{2}\right)-\varepsilon\left(\boldsymbol{q}_{1}\right)-\varepsilon\left(\boldsymbol{q}_{2}\right)\right) \\
& \text { with } \quad \cdot B d^{3} p_{1} d^{3} p_{2} d^{3} p_{2}^{\prime} d^{3} p_{1}^{\prime}=0
\end{aligned}
$$

$$
B=\varrho\left(\boldsymbol{p}_{1}\right) \varrho\left(\boldsymbol{p}_{2}\right)\left(1-\varrho\left(\boldsymbol{q}_{2}\right)\right)\left(1-\varrho\left(\boldsymbol{q}_{1}\right)\right)-\left(1-\varrho\left(\boldsymbol{p}_{1}\right)\right)\left(1-\varrho\left(\boldsymbol{p}_{2}\right) \varrho\left(\boldsymbol{q}_{2}\right) \varrho\left(\boldsymbol{q}_{1}\right)\right) .
$$

Since the test functions are essentially arbitrary $B$ has to vanish whenever $\varepsilon\left(\boldsymbol{p}_{1}\right)+\varepsilon\left(\boldsymbol{p}_{2}\right)-\varepsilon\left(\boldsymbol{q}_{2}\right)-\varepsilon\left(\boldsymbol{q}_{1}\right)=0$. This means that

$$
R(\boldsymbol{p})=\log \left(\frac{1-\varrho(\boldsymbol{p})}{\varrho(\boldsymbol{p})}\right)
$$

has to be a linear function of $\varepsilon(\boldsymbol{p})$ i.e.

$$
\varrho(\boldsymbol{p})=\frac{1}{1+e^{\alpha+\beta \varepsilon(\boldsymbol{p})}}
$$

with $\alpha, \beta$ two real parameters. This the Fermi distribution.

Acknowledgements. The authors greatfully acknowledge discussions with H. Araki, A. Connes, and M. Takesaki. They are indebted to II. Institut für Theoretische Physik, Universität von Hamburg, and Centre Universitaire de Marseille-Luminy, Université d'Aix-Marseille II, for financial support. 


\title{
References
}

1. Kubo, R.: J. Physic. Soc. Japan 12, 570 (1957)

2. Martin, P.C., Schwinger,J.: Phys. Rev. 115, 1342 (1959)

3. Haag, R., Hugenholtz, N., Winnink, M.: Commun. math. Phys. 5, 215 (1967)

4. Hugenholtz, N.: In: Instructional Conf. London Math. Soc. August 23th-Sept. 11th (1971)

5. Cargèse lectures in physics, Vol. 4. New York: Gordon and Breach 1970

6. Takesaki, M.: Lecture Notes in Math., Vol. 128. Berlin-Heidelberg-New York: Springer 1970

7. Varenna lectures on $C^{*}$-algebras and physical applications. Italian Physical Society (1973)

8. Trych-Pohlmeyer,E.B.: Forthcoming thesis Hamburg University 1974

9. Ruelle, D.: Statistical mechanics. Rigorous Results. New York: Benjamin 1969

10. Borchers, H.J.: Commun. math. Phys. 2, 49 (1966)

11. Kastler,D., Pool,J.C.T., Thue Poulsen,E.: Commun. math. Phys. 12, 175 (1969)

12. Araki,H.: Publ. RIMS. Kyoto University 9, No. 1 (1973); see also Araki in 7

13. Araki,H.: Ann. Sci. Ecole Norm. Sup. 6, No. 1 (1973)

14. Yosida, K.: Functional analysis. Berlin-Heidelberg-New York: Springer 1965

15. Robinson, D.W. in [7] and: Commun. math. Phys. 31, 171 (1973)

16. Ruelle, D.: Commun. math. Phys. 3, 133 (1966)

17. Doplicher, S., Kastler, D., Robinson, D. W.: Commun. math. Phys. 3, 1 (1966)

18. Doplicher,S., Kadison,R.V., Kastler,D., Robinson,D.W.: Commun. math. Phys. 6, 101 (1967). See also E. Störmer in [5]

19. Wightman,A.S.: Proc. Int. Congress of Mathematicians (14-23. Aug. 1962)

20. Borchers, H.J.: Commun. math. Phys. 1, 281 (1965)

21. Borchers, H.J.: Nachr. Akad. Wiss. Göttingen II 2, 1 (1973)

22. Arveson, W.: On groups of automorphisms of operators algebras. (Preprint)

23. Connes, A.: Ann. Sci. Ecole Norm. Sup. 6, 18 (1973)

24. Störmer, E.: Commun. math. Phys. 28, 279 (1972)

Communicated by J. L. Lebowitz

\author{
R. Haag \\ II. Institut für Theoretische Physik \\ der Universität \\ D-2000 Hamburg 50 \\ Luruper Chaussee 149 \\ Federal Republic of Germany \\ D. Kastler \\ E. B. Trych-Pohlmeyer \\ Département de Physique \\ Centre Universitaire de Marseille-Luminy \\ Marseille, France
}


